\title{
Assessment of two methods for gravity field recovery from GOCE GPS-SST orbit solutions
}

\author{
K. Arsov ${ }^{1}$ and R. Pail ${ }^{2}$ \\ ${ }^{1}$ Austrian Academy of Sciences, Space Research Institute, Dept. of Satellite Geodesy, Schmiedlstrasse 6, A-8042 Graz, \\ Austria \\ ${ }^{2}$ Graz University of Technology, Institute of Geodesy, Dept. of Theoretical Geodesy, Steyrergasse 30, A-8010 Graz, Austria
}

\begin{abstract}
In the course of the GOCE satellite mission, the high-low Satellite to Satellite Tracking (SST) observations have to be processed for the determination of the long wavelength part of the Earth's gravity field. This paper deals with the formulation of the high-low SST observation equations, as well as the methods for gravity field recovery from orbit information. For this purpose, two approaches, i.e. the numerical integration of orbit perturbations, and the evaluation of the energy equation based on the Jacobi integral, are presented and discussed. Special concern is given to the numerical properties of the corresponding normal equations. In a closed-loop simulation, which is based on a realistic orbit GOCE configuration, these methods are compared and assessed. However, here we process a simplified case assuming that non-conservative forces can be perfectly modelled. Assuming presently achievable accuracies of the Precise Orbit Determination (POD), it turns out that the numerical integration approach is still superior, but the energy integral approach may be an interesting alternative processing strategy in the near future.
\end{abstract}

Key words. High-low SST - gravity field - GOCE - variational equations - least squares adjustment

\section{Introduction}

Based on the assumption that precise GOCE orbits are available from a POD solution, several strategies for the recovery of the harmonic coefficients exist:

- Numerical integration of orbit perturbations (classical concept);

- Evaluation of the energy equations based on the Jacobi integral;

- Derivation of satellite accelerations.

Correspondence to: K. Arsov (kirco.arsov@oeaw.ac.at)
In this paper, as the baseline method we follow the classical approach, i.e. the numerical integration of orbit perturbations. First we will give a short summary of the method that is used for the gravity field recovery based on SST observations, implemented as the software module ARCSST. The formulation as well as the structure of the linearized observation equations model will be presented. Additionally, we will give an overview of the numerical integration model, together with some performance tests. Since the classical SST concept is based on linear orbit perturbations with respect to a reference orbit, the partials of the position with respect to the spherical harmonic coefficients are needed. In the sequel their derivation, by means of numerical integration of the variational equations, will be explained. A case study analyzing the influence of the arc length as well as numerical investigations on the linearization errors will be presented. Concerning the estimation of the static coefficients, a numerical simulation will be introduced, where we determine the spherical harmonic coefficients up to degree and order 80. For this purpose, an orbit with a 59 days repeat period which is close to the planned GOCE satellite orbit is integrated numerically, and the unknown spherical harmonic coefficients are determined in the noise-free case. Furthermore, another configuration will be presented, where orbital noise based on a simulated kinematic POD is introduced in the vector of measurements by properly taking into account the variance-covariance information of the simulated orbit. Additionally, some considerations on the orbital energy balance principle will be given. This second technique of harmonic coefficients estimation, which is based on the Jacobi integral approach, will be presented and assessed.

\section{Orbit perturbations of one satellite}

The concept to evaluate the SST information for gravity field determination we perform as the baseline strategy is the potential coefficients determination using the orbit perturbations of one satellite (SST in high-low mode). The 
Low Earth Orbiter (LEO) satellite has an altitude of about $250 \mathrm{~km}$, and is tracked by a at least 7 GPS satellites (Arsov et al., 2002) flying at a high altitude of about $20200 \mathrm{~km}$. In the process of the POD the coordinates of the LEO satellite can be determined with a high accuracy of some centimeters. If we compare the model (standard) orbit, which is based on a priori gravity field information, with the GOCE orbit determined by GPS, generally they will not coincide, because of the imperfection of our model. The goal is to improve the a priori gravity model (e.g. EGM96) using the information from the orbit perturbations. It should be mentioned, that as additional unknowns we have to introduce also the initial satellite state vector (position and velocity unknowns) representing the 6 integration constants. The adjustment has to be performed iteratively, where the standard orbit is updated after each adjustment step for the coordinate differences of the initial epoch, and of course for the unknown geopotential coefficients. The observational equations based on linear satellite orbit perturbations are given as:

$\Delta \boldsymbol{\rho}=\mathbf{W} \delta \boldsymbol{\beta}+\boldsymbol{\Phi} \delta \boldsymbol{x}$

where $\Delta \boldsymbol{\rho}$ represents the residuals from the standard orbit for each epoch expressed in the inertial system by the coordinates $X, Y$ and $Z, \delta \boldsymbol{x}$ represents the initial state vector to be determined, and $\delta \boldsymbol{\beta}$ represents the unknown potential coefficient differences. $\mathbf{W}$ is the parameter sensitivity matrix, whose elements are given as the derivatives of the orbit positions $\boldsymbol{r}$ with respect to the coefficients $\boldsymbol{\beta}$ :

$\mathbf{W}=\frac{\partial \boldsymbol{r}}{\partial \boldsymbol{\beta}}$

The state transition matrix $\boldsymbol{\Phi}$ (with 18 elements, if we use only the position discrepancies as measurements) is given as

$\boldsymbol{\Phi}=\left\{\frac{\partial \boldsymbol{r}}{\partial \boldsymbol{r}_{0}}, \frac{\partial \boldsymbol{r}}{\partial \dot{\boldsymbol{r}}_{0}}\right\}$

The components of $\boldsymbol{\Phi}$ are evaluated using the standard orbit positions. In our concept, the SST data are processed using a multi-arc method, where the vector of unknowns $\delta \boldsymbol{x}$ is composed of the initial positions and velocities for each arc. The major computational effort in evaluating Eq. (1) is the computation of the parameter sensitivity matrix $\mathbf{W}$. In our software developments for the numerical integration of the orbit and variational equations, we closely follow Cunningham (1970), but with some modifications that enable us to work with normalized spherical harmonic coefficients.

For the orbit and variational equations integration, two routines were implemented: the Runge-Kutta 5 (in the further context abbreviated by RK5) and the Gauss-Jackson 14 (GJ14) predictor-corrector method. Since in our case the GJ14 needs 13 initial values, we use the RK5 integration to obtain these 13 values, and subsequently the integration is continued applying GJ14.

RK5 can achieve the accuracy of a Taylor series, truncated after the linear term, without requiring the calculation of derivatives beyond the first. Many variations of the RungeKutta method exist. In the course of this study we apply Butcher's fifth order method (cf. Boulet, 1991). The GJ14 predictor-corrector method, or second sum method for numerical integration, is a powerful method for the integration of the second order differential equations. Here, we shall not give the complete derivations of GJ14. More details about the derivation itself can be found in Montenbruck (2000). In order to test the implementation of the GJ14 routine and to analyze its performance, a small test run was performed. It is known that the equations of satellite motion, using a point mass as the only gravitational attraction, can be described analytically by the Keplerian equations. Considering our GJ14 and RK5 routines, the same scenario could be achieved by assigning zero values to all harmonic coefficients excepting the term $C_{00}$. We perform an orbit integration of 1 year both with Keplerian equations and the GJ14 integrator, and in Fig. 1a the high accuracy of the GJ14 integrator can be noticed, yielding discrepancies of about $10 \mathrm{~cm}$ after the 1 year integration period.

Another test of the numerical stability can be performed by the use of the energy conservation law. It is known that in a closed system the sum of the potential and the kinetic energy is constant. Applied to our satellite motion, this law reads (Jekeli, 1999):

$V+\sum_{k} \int_{t_{0}}^{t} \boldsymbol{F}_{k} \dot{\boldsymbol{r}_{k}} d t-\frac{1}{2}|\dot{\boldsymbol{r}}|^{2}-\omega(X \dot{Y}-Y \dot{X})=\mathrm{const}$

In Eq. (4), $V$ represents the Earth's geopotential, the second term is the energy dissipation term (contribution of the non-conservative forces), the third term represents kinetic energy, whereas the last term is due to the coordinate transformation between the inertial and Earth-fixed system. If the Earth's gravity field were rotationally axial-symmetric, this last term would be 0 . In Eq. (4) only the geopotential is computed in the Earth-fixed coordinate system, whereas all other terms have to be evaluated in the inertial system.

At this point it should be mentioned, that in our computations, the second term in Eq. (4) is set to 0, i.e. assuming that no non-conservative forces are acting on the satellite. In this test run the geopotential used in Eq. (4) is the OSU91a model complete up to degree and order 180. Figure $1 \mathrm{~b}$ presents the deviations from the constant, which is in the order of $10^{-5} \mathrm{~m}^{2} \mathrm{~s}^{-2}$, for a one year integration period. The relative accuracy in the order of $10^{-13}$ demonstrates the high precision of the GJ14 integrator.

\section{Harmonic coefficient estimation}

\subsection{Numerical integration of orbit perturbations}

In the following we are going to recover the spherical harmonic coefficients based on the numerical integration of the orbit and variational equations. Before proceeding with the actual computations, we will perform a small test in order to assess the linearization errors and their influence on the 

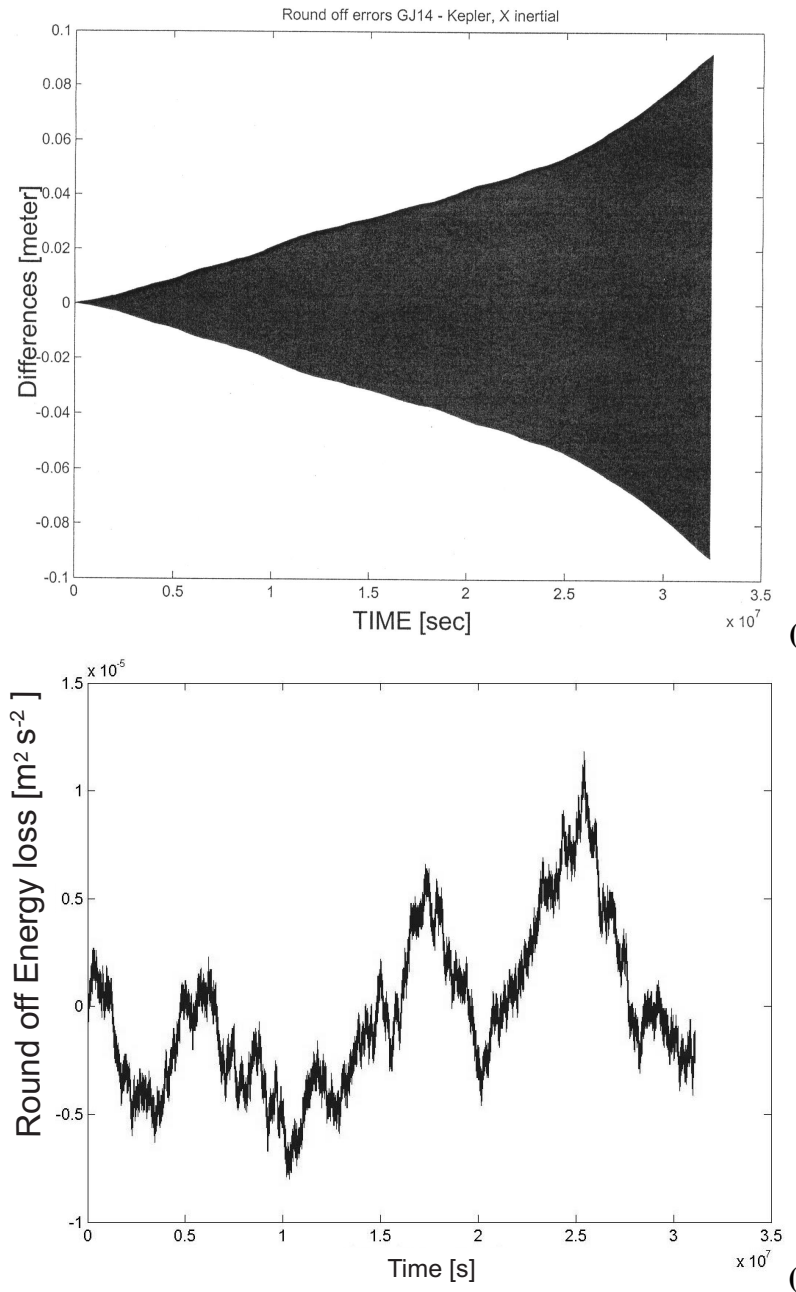

(a)

Fig. 1. (a) Position accuracy $[\mathrm{m}]$ and (b) energy loss $\left[\mathrm{m}^{2} \mathrm{~s}^{-2}\right]$ of GJ14.

normal equations matrix. Additionally, we will perform a small experiment to see which arc length should be used in the further computations by highlighting the linearization errors introduced in different scenarios.

The basic linear model for the observational equations as defined in Eq. (1) for each arc is used. Since we are only interested in the spherical harmonic coefficients $\delta \boldsymbol{\beta}$, the additional parameters ( 6 position and velocity initial conditions parameters per arc) have to be eliminated properly for each arc. In general, the normal equation matrix is a full matrix. It is given for one arc as (Visser et al., 2001):

$$
\left[\begin{array}{ll}
\boldsymbol{N}_{x x} & \boldsymbol{N}_{x \beta} \\
\boldsymbol{N}_{\boldsymbol{\beta} x} & \boldsymbol{N}_{\boldsymbol{\beta} \beta}
\end{array}\right]\left(\begin{array}{l}
\delta \boldsymbol{x} \\
\delta \boldsymbol{\beta}
\end{array}\right)=\boldsymbol{R}=\left(\begin{array}{l}
\boldsymbol{R}_{\boldsymbol{x}} \\
\boldsymbol{R}_{\boldsymbol{\beta}}
\end{array}\right)
$$

and the elimination of the initial state vector yields the reduced normal equation matrix for this arc:

$\left[\boldsymbol{N}_{\beta \beta}-\boldsymbol{N}_{\boldsymbol{\beta} x} \boldsymbol{N}_{x x}^{-1} \boldsymbol{N}_{x \beta}\right] \delta \boldsymbol{\beta}=\boldsymbol{R}_{\beta}-\boldsymbol{N}_{\beta x} \boldsymbol{N}_{x x}^{-1} \boldsymbol{R}_{x}$

where $N$ denotes the normal equation matrix, $\delta \boldsymbol{\beta}$ represents the unknown spherical harmonic coefficients, $\delta \boldsymbol{x}$ denotes the

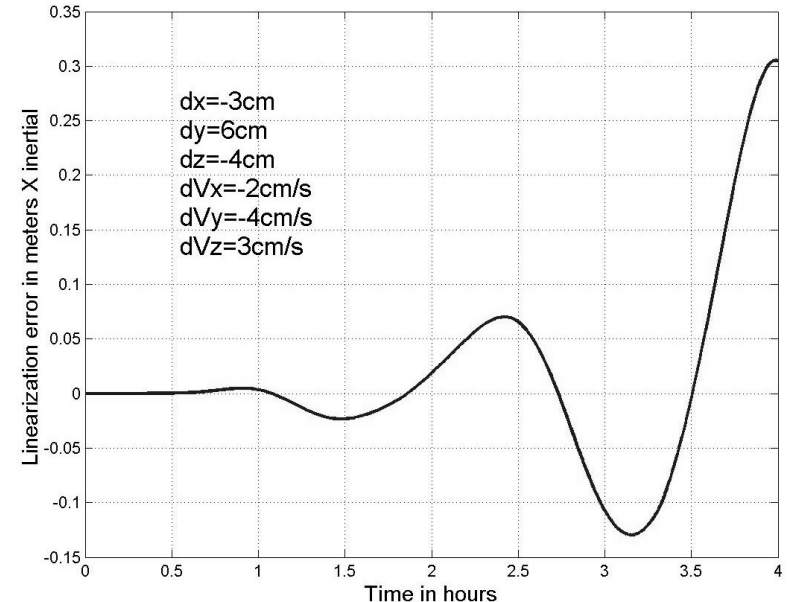

(a)

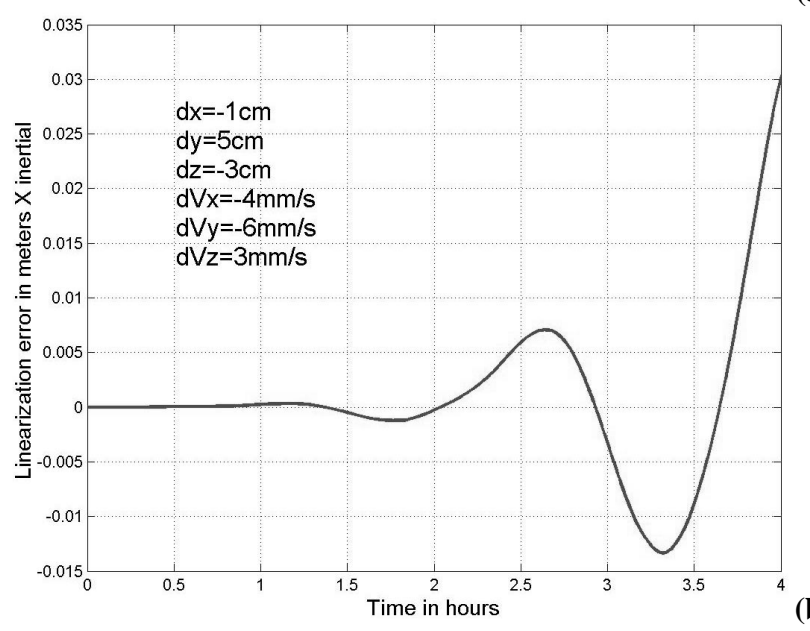

(b)

Fig. 2. Linearization error for different initial conditions unknowns: (a) velocity errors $\sim \mathrm{cm}$; (b) velocity errors $\sim \mathrm{mm}$.

initial state vector and $\boldsymbol{R}$ is the right-hand side. The final normal equation matrix is obtained by summation of the reduced normal equations and the reduced right-hand sides of each arc.

Now, we investigate the influence of the unknown initial conditions on the linearization errors in the SST arc-wise computation, using a 10-days orbit with a sampling rate of $5 \mathrm{~s}$, an inclination of $i=96.6^{\circ}$, and a nominal height of about $250 \mathrm{~km}$. The spherical harmonic coefficients were included in the orbit up to degree and order 50. At first the standard orbit was computed applying the OSU91a model, and in the second run, the EGM96 model was used as the "true" gravity field. Furthermore, in the initial conditions, discrepancies in the $\mathrm{cm}$ and $\mathrm{mm}$ range were introduced. If we again take a look at our basic observation model as given in Eq. (1), this means that now we know the right-hand side, and by inserting these values into the observation model, we can assess the sensitivity of our observational model with respect to errors in the initial values, and with respect to the 

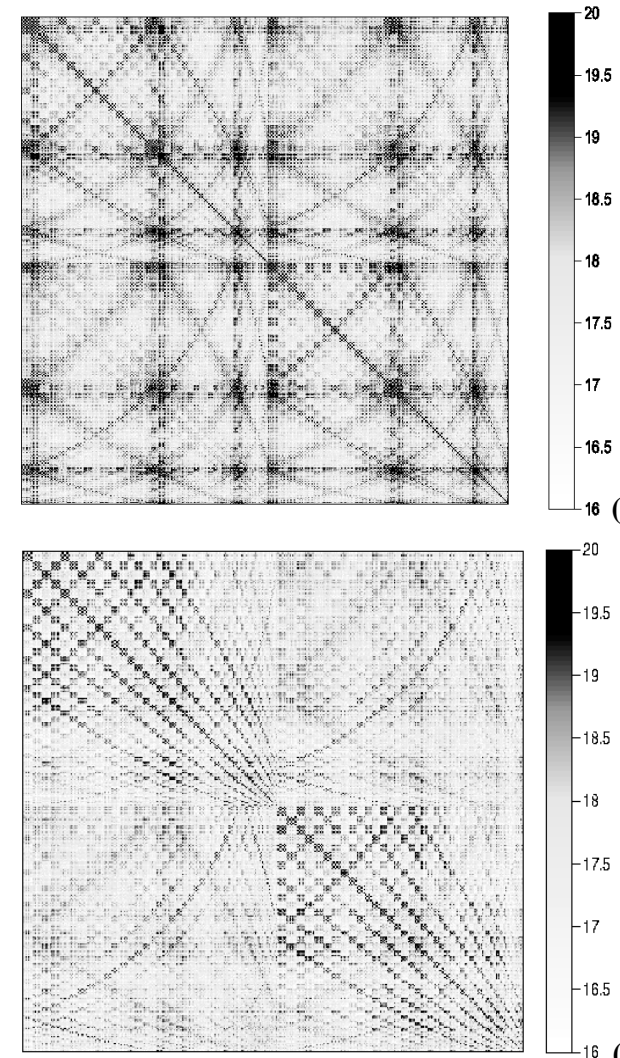

(a) cond $=5.0 \mathrm{E}+009$
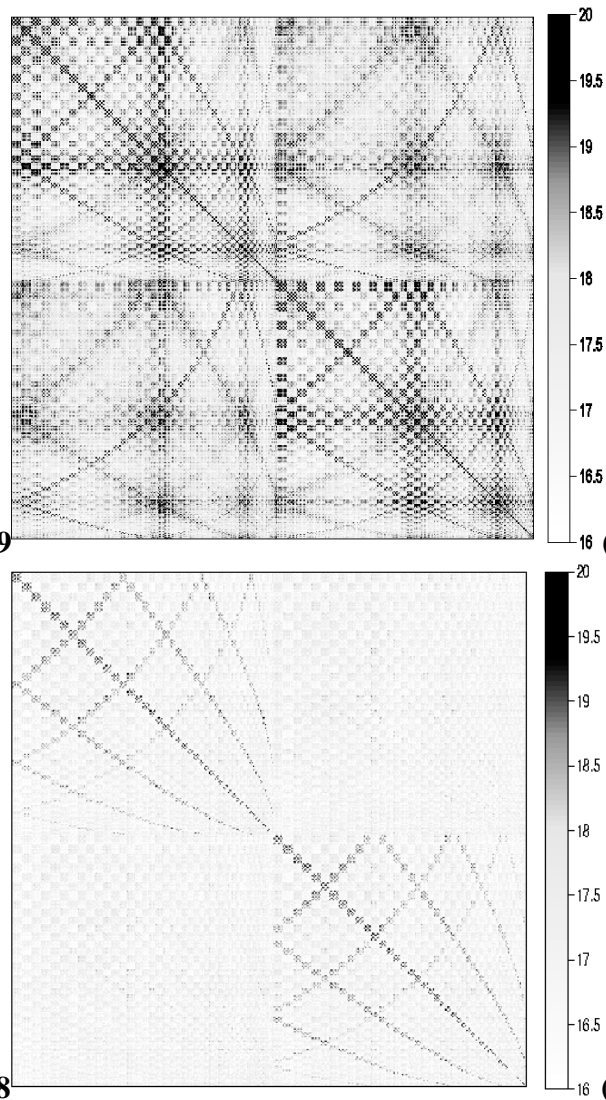

(d) cond $=2.7 \mathrm{E}+007$

Fig. 3. Normal equation matrices for arc lengths (a) $18 \mathrm{~h}$; (b) $12 \mathrm{~h}$; (c) $6 \mathrm{~h}$; (d) $2 \mathrm{~h}$ for the degree 50 solution. Corresponding coefficients sorted by harmonic order $m, C_{l m}$ and $S_{l m}$ separated $\left(C_{2,0}, C_{3,0}, C_{4,0}, \ldots, C_{50,0}, C_{2,1}, C_{3,1}, C_{4,1}, \ldots, C_{50,50}, S_{2,1}, S_{3,1}, S_{4,1}, \ldots\right)$.

spherical harmonic coefficients. We observe, that if we keep the initial condition unknowns at 0 , almost no influence on the linearization errors resulting from OSU91a and EGM96 model differences can be observed, because they match quite well. On the other hand, introducing the initial condition unknowns may highly influence the linearization errors of the SST model. Therefore, we expect to obtain a big correlation between the initial conditions and the harmonic coefficients.

Figure 2 presents the obtained results for different values of the initial conditions unknowns. In Fig. 2a the initial conditions are inaccurate at the $\mathrm{cm}$ level and the initial velocity errors are in the order of several $\mathrm{cm} / \mathrm{s}$, and consequently the linearization errors after $4 \mathrm{~h}$ are about $\pm 30 \mathrm{~cm}$, which is considerably large, having in mind the accuracy of the POD of a few $\mathrm{cm}$. However, after about $2 \mathrm{~h}$, they are within $\pm 3 \mathrm{~cm}$, which may give us a better understanding on choosing the right arc length in our further computations. Actually, Fig. 2a represents the realistic errors in the initial conditions, if we assume that the positions coming from the POD have a rms error of about $3 \mathrm{~cm}$. If we reduce the initial conditions unknowns of the velocities to a couple of $\mathrm{mm} / \mathrm{s}$, keeping the position errors of the initial conditions at the cm-level (Fig. 2b), we have a slightly improved scenario, but it should be mentioned that in practice velocity accuracies within a couple of $\mathrm{mm} / \mathrm{s}$ are difficult to obtain. Based on the results in Fig. 2 we decided to use arc lengths of two hours for our further SST tests. It is also interesting to see how the linearization errors affect the normal equations matrix. For this purpose, we performed another test with the orbit configuration mentioned above, where different arc lengths were used in the spherical harmonics estimation procedure. The initial conditions unknowns were kept in the the same order as in Fig. 2a.

Figure 3a demonstrates, that in the case of $18 \mathrm{~h}$ arcs a strong correlation of the initial state vector unknowns with the harmonic coefficients exists. The ordering scheme of the normal equations is by harmonic order $m$, leading to a dominant block-diagonal structure, but also horizontal and vertical off-diagonal bands can be distinguished. These resonance bands are a system-intrinsic physical phenomenon, and they occur close to the orbit resonance orders at 0,16 and 32 , which represent the integer multiples of the orbital revolutions per day. These resonances decrease as the arc length decreases, and in Fig. 3d almost no resonance frequencies can be observed. Also, we notice the decrease in the condition number of the normal equations as the arc length decreases. This again suggests to use $2 \mathrm{~h}$ arcs in our future simulations. 


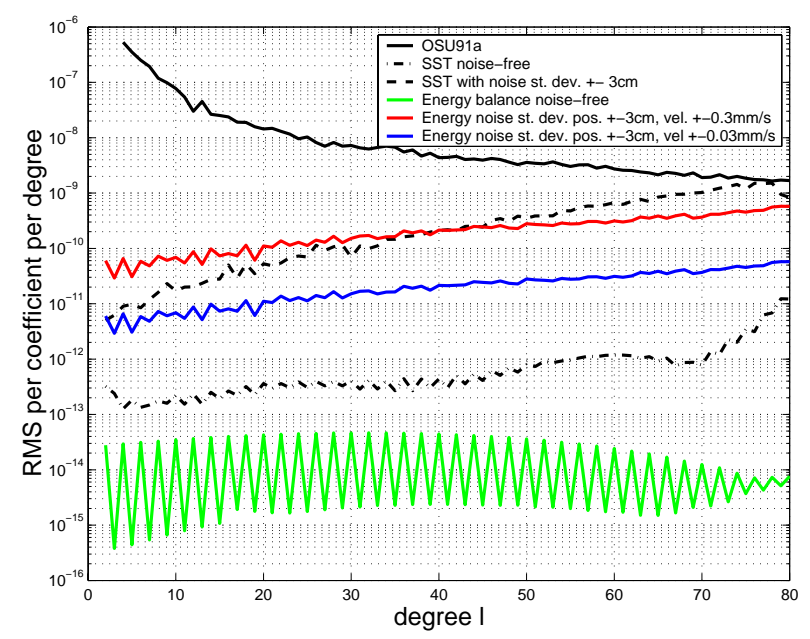

Fig. 4. Degree rms plots for different SST solutions.

\subsection{Energy integral approach}

As an alternative spherical harmonics estimation procedure, we may also use Eq. (4), rewritten in the form (cf. e.g. Han et al., 2002)

$V+$ const $=\frac{1}{2}|\dot{\boldsymbol{r}}|^{2}-\sum_{k} \int_{t_{0}}^{t} \boldsymbol{F}_{k} \dot{\boldsymbol{r}_{k}} d t+\omega(X \dot{Y}-Y \dot{X})$

It is obvious, that by application of the energy conservation law the harmonic coefficients can be obtained from the orbit coordinates. We will use also Eq. (7) for spherical harmonics estimation in the further context, since the model is linear and immediately applicable to the orbit coordinates and velocities. Compared with the classical numerical integration method, one of the major advantages of the energy integral approach is the absence of resonance phenomena in the normal equations.

\subsection{Simulation and results}

In order to assess the two solution techniques with respect to the CPU time and obtainable accuracy, we performed the harmonic coefficients estimation applying both the numerical integration of the variational equations and the energy integral methods. This simulation is based on a sun-synchronous 59 days repeat orbit with a nominal altitude of $250 \mathrm{~km}$, including the gravity field complete up to degree and order 80 and a sampling rate of $5 \mathrm{~s}$.

The results in terms of the degree RMS are presented in Fig. 4. From these plots, one can deduce, that in the noisefree scenario the energy balance approach (green curve) shows a better accuracy than the numerical integration technique (black curve). A major difference is the run-time, which is in the present configuration approximately 20 times longer for the numerical integration approach. Therefore, the ARCSST routine for the estimation of the harmonic coefficients based on the numerical integration approach was adapted to allow run on a parallel platform (Graz Beowulf cluster, cf. Plank, 2002).

For a more realistic scenario including measurement noise, a realistic orbital error description in terms of propagated position variance/covariance matrices together with the corresponding position noise distributions was provided by TU Munich, where POD simulation was carried out based on the kinematic POD approach (cf. Arsov et al., 2002).

As far as the energy balance approach is concerned, we need both position and velocity noise information. If we assume that velocities are derived as position differences, a simple orbital energy propagation reveals that the major error term is related to the kinetic energy, and thus we can conclude that velocity errors play a dominant role in these error considerations. The energy observation error $\sigma_{o b s}$ can be approximated by

$\sigma_{o b s} \approx|\dot{\boldsymbol{r}}| \sigma_{|\dot{\boldsymbol{r}}|} \approx|\dot{\boldsymbol{r}}| \frac{\sqrt{2}}{\Delta t} \sigma_{\text {pos }} \sqrt{1-\rho}$

where $\sigma_{|\dot{r}|}$ is the velocity error, which can be roughly described in terms of an error propagation of the orbit position error $\sigma_{p o s}$. If the velocity is computed from orbit positions, its accuracy mainly depends on the correlation $\rho$ of adjacent positions and on the sampling interval $\Delta t$. However, it should be noticed that the interpolation error, which occurs when the velocities are derived from the positions, is counteracting.

Concerning the numerical integration method, in our second run the noise of $3 \mathrm{~cm}$ rms in position provided by TU Munich was superposed, and the corresponding variance/covariance information was included in the stochastic model. The dashed black curve in Fig. 4 shows the degree rms of the deviations of the numerical integration solution from the initial OSU91a model.

As far as the energy conservation approach is concerned, the same position noise data was used as in the numerical integration approach, and the corresponding velocity noise was obtained by error propagation. Assuming position errors in the order of a few $\mathrm{cm}$ leads in the optimum case to velocity errors in the order of $10^{-3}$ to $10^{-4} \mathrm{~m} / \mathrm{s}$. From Fig. 4 we can also deduce that in order to obtain roughly the same accuracy as the numerical integration, the velocities have to be known with an accuracy of at least $10^{-4} \mathrm{~m} / \mathrm{s}$ (red curve), and even then we have a lower accuracy as the numerical integration approach in the spectral region up to degree 35 .

\subsection{Summary and discussion}

In this paper two methods of the determination of the Earth's gravity field from the high-low SST observations were introduced and discussed: the numerical integration of the orbit and the variational equations, and the energy integral approach. In order to assess these methods, a closed-loop simulation, based on a realistic GOCE orbit configuration, was performed. The chosen orbit contains harmonic coefficients parameterizing the Earth's gravity field complete up to degree and order 80, and they are recovered applying 
both methods. The harmonic coefficients estimation was performed both in the noise-free case and including a realistic noise, coming from the simulated GOCE POD, with the simplifying assumption that the non-conservative forces can be perfectly modelled.

Summarizing these simulations, it can be concluded, that the energy balance approach has several advantages, such as strictly linear observation equations, or the absence of resonance effects, and it requires considerably less CPU time than the numerical integration method. However, the accuracy of the coefficient estimates depends to a great extent on the noise amplitudes of the observations, and it could be demonstrated, that the energy balance method is highly sensitive to velocity errors. Assuming presently achievable POD accuracies, the numerical integration yields superior results concerning the accuracy of the estimated coefficients. The normal equation matrix is numerically regular, which is partly due to the short arc lengths of $2 \mathrm{~h}$. The noise of $3 \mathrm{~cm}$ rms in the orbit degrades the accuracy of the spherical harmonics coefficients by about two significant digits compared to the noise-free scenario.

Acknowledgements. This study was performed in the course of the GOCE project "From Eötvös to mGal+", funded by the European Space Agency (ESA) under contract No. 14287/00/NL/DC.

\section{References}

Arsov, K., Badura, T., Höck, E., Pail, R. Rothacher, M., and Švehla, D.: Effect of Temporal Variations on High-low SST observations, ESA project 'From Eötvös to mGal +', Draft Final Report, ESA/ESTEC Contract 14287/00/NL/DC, European Space Agency, 217-316, 2002.

Boulet, D.: Methods of orbit determination for the microcomputer, 564 p., Willmann-Bell, Inc., Richmond, Virginia, USA, 1991.

Cunningham, E. L.: On the computation of the spherical harmonic terms needed during the numerical integration of the orbital motion of an artificial satellite, Celestial Mechanics, Vol. 2, pp. 207 216, 1970.

Han, S.-C., Jekeli, C., and Shum, C. K.: Efficient gravity field recovery using in-situ disturbing potential observables from CHAMP, Geophys. Res. Lett., 29, 16, 36/1-36/4, 2002.

Jekeli, C.: The determination of gravitational potential differences from satellite-to-satellite tracking, Celest. Mech. Dyn. Astron., Kluwer Academic Publishers, Vol. 75, 85-101, 1999.

Montenbruck, O. and Gill, E.: Satellite Orbits, Models, methods, applications, Springer-Verlag Berlin Heidelberg, New York, 2000.

Plank, G.: Implementation of the PCGMA-package on massive parallel systems, ESA project 'From Eötvös to mGal +', Draft Final Report, ESA/ESTEC Contract 14287/00/NL/DC, European Space Agency, pp. 183-214, 2002.

Visser, P. N. A. M., van den IJssel, J., Koop, R., and Klees, R.: Exploring gravity field determination from orbit perturbations of the European Gravity Mission GOCE, J. Geodesy, 75, 89-98, 2001 . 